\title{
Effects of fruit and vegetables intake in periodontal diseases: A systematic review
}

\section{Wpływ spożywania owoców i warzyw na przebieg chorób przyzębia - systematyczny przegląd piśmiennictwa}

\author{
Aleksandra Skoczek-Rubińska ${ }^{A-D}$, Joanna Bajerska ${ }^{\mathrm{E}, \mathrm{F}}$, Katarzyna Menclewicz ${ }^{\mathrm{B}-\mathrm{D}}$ \\ Institute of Human Nutrition and Dietetics, Poznan University of Life Sciences, Poland \\ A - research concept and design; B - collection and/or assembly of data; $C$ - data analysis and interpretation; \\ $D$ - writing the article; $E$ - critical revision of the article; $F$ - final approval of the article
}

Address for correspondence

Aleksandra Skoczek-Rubińska

E-mail: aleksandra.skoczek-rubinska@up.poznan.pl

\section{Funding sources}

None declared

Conflict of interest

None declared

Received on February 27, 2018

Reviewed on August 28, 2018

Accepted on 0ctober 19, 2018

Published online on December 27, 2018

Cite as

Skoczek-Rubińska A, Bajerska J, Menclewicz K. Effects of fruit and vegetables intake in peridontal diseases: A systematic review. Dent Med Probl. 2018;55(4):431-439. doi:10.17219/dmp/99072

D0I

10.17219/dmp/99072

Copyright

๑) 2018 by Wroclaw Medical University

and Polish Dental Society

This is an article distributed under the terms of the

Creative Commons Attribution Non-Commercial License

(http://creativecommons.org/licenses/by-nc-nd/4.0/)

\begin{abstract}
Periodontal diseases affect up to $90 \%$ of the population worldwide. Deficiencies in vitamins, minerals and polyphenolic compounds, whose main sources are fruit and vegetables (F\&V), may predispose to these diseases. The PICO (Patient, Intervention of interest, Comparison or Control Intervention, and Outcome) question was: What is the effect of F\&V intake on the outcomes of periodontal diseases, observed in either experimental or observational studies of human populations suffering from periodontitis or gingivitis, compared to controls without intervention or healthy people.

The review was conducted using the Preferred Reporting Items for Systematic Reviews and Meta-Analyses (PRISMA) protocol for search strategy, selection criteria and data extraction. The searched databases included MEDLINE (PubMed), Scopus and Google Scholar. A total of 181 potentially relevant articles were identified, which were then evaluated according to pre-specified criteria relating to relevance and quality. Finally, 15 articles were selected for review. Four articles described intervention studies, 3 reported on prospective and retrospective cohort studies, and 8 concerned cross-sectional studies. A total of 10,604 people aged 15-90 years took part in the studies. On the basis of the research it can be concluded that the consumption of at least 5 servings of F\&V per day may prevent the progression of periodontal diseases, especially periodontitis, and even tooth loss. Moreover, incorporation of specific F\&V into the diet or, alternatively, their lyophilized forms, and nutritional education activities seem to support the standard of care therapy of gingivitis and periodontitis. However, further observational and well-designed experimental studies, with homogeneous periodontal status outcomes, are needed to confirm these findings. Furthermore, professional dietetics care for periodontal patients should become an integral component of the healthcare program.
\end{abstract}

Key words: diet, fruit, vegetables, periodontal diseases

Słowa kluczowe: dieta, owoce, warzywa, choroby przyzębia 


\section{Introduction}

Periodontal diseases refer to a range of serious pathological conditions that occur in the tissues surrounding the teeth in response to bacterial accumulation or dental plaque. It is estimated that they affect as much as $90 \%$ of the population worldwide, and almost half of the world's population suffers from periodontitis. ${ }^{1-3}$ The classification established during the 2017 International Workshop for a Classification of Periodontal Diseases and Conditions distinguishes dental biofilm-induced gingivitis, non-plaque-induced gingival diseases and conditions, necrotizing periodontal diseases, periodontitis as a manifestation of systemic diseases, forms of the periodontal disease previously recognized as 'chronic' or 'aggressive', now grouped under a separate category - 'periodontitis', and progressive and acquired deformities and conditions. ${ }^{4}$ A significant role in the pathogenesis of periodontal diseases is attributed to the formation of bacterial biofilm on tooth surfaces, i.e., dental plaque. ${ }^{2,5}$ However, what triggers the progression of inflammatory conditions is the disturbance of the equilibrium between the pathogenic bacteria attacking the periodontal tissue and the host's defense mechanisms. The severity and progression of the diseases is a result of numerous factors, including genetic predisposition, age, general health condition, and improper nutrition. It has also been observed that the prevalence of periodontitis is greater in smokers, less educated individuals and those of a lower socioeconomic status. ${ }^{6}$ Untreated periodontitis disturbs the process of mastication and may lead to partial or total tooth loss. ${ }^{7,8}$ Problems associated with the mastication of food affect the composition of the diet and result in further deterioration of the patient's health condition. It has been confirmed that patients suffering from periodontitis have higher rates of diabetes, cardiovascular disease and cerebral stroke, with the risk increasing with age. ${ }^{9,10}$ Typically, patients seek medical attention only in situations requiring dental intervention or pharmacological treatment. However, next to adequate oral hygiene, proper diet composition seems to be an effective measure to prevent the diseases or even to modulate an already existing condition. ${ }^{11}$ It has been confirmed that deficiencies in several nutrients, such as vitamins and minerals, strongly enhance the progression of periodontal diseases. ${ }^{12,13}$ The primary sources of these important nutrients include fruit and vegetables $(\mathrm{F} \& \mathrm{~V})$. A PICO (Patient, Intervention of interest, Comparison or Control Intervention, and Outcome) question was formulated to identify key words that would drive the literature search. ${ }^{14}$ The PICO question was: What is the effect of F\&V intake on the outcomes of periodontal diseases, observed in either experimental or observational studies of human populations suffering from periodontitis or gingivitis, compared to controls without intervention or healthy people.

\section{Search strategy}

The MEDLINE (PubMed), Scopus and Google Scholar electronic databases were searched to find articles that might answer the above question. The cutoff date for this search was December 15, 2017. The search was limited to studies on human subjects, published between 1990 and 2017, in English, and available in full text. The search terms were: [periodontitis OR periodontal OR chronic periodontitis OR periodontal disease OR periodontal diseases OR aggressive periodontitis] AND [fruit OR fruits OR vegetable OR vegetables] AND [longitudinal OR studies OR supplementation OR consumption OR prospective studies OR cohort studies OR randomized clinical trial OR cross-sectional studies OR intervention]. No other restrictions were established, and the free-text strategy and Medical Subject Headings (MeSH) terms were applied in the search process. This systematic review was conducted in line with the Preferred Reporting Items for Systematic Reviews and Meta-Analyses (PRISMA) statement. $^{15}$

\section{Study selection and eligibility criteria}

The study selection was conducted independently by 2 reviewers (ASR, KM), with any disagreements resolved by the $3^{\text {rd }}$ reviewer (JB). The eligibility criteria were formed based on the study question. This systematic review considered only original studies with the following designs: randomized controlled trials (RCTs), and cohort and cross-sectional studies on the effect of F\&V intake on gingivitis and periodontitis patients. We also decided to exclude descriptive observational studies (e.g., case studies), reviews, systematic reviews with and without meta-analysis, letters, book chapters, reports, studies without an abstract or where the full text was not available, research conducted on cells, research conducted on nonhuman species, studies conducted only among healthy people or among patients with oral diseases other than gingivitis and periodontitis (e.g., caries), and studies evaluating the effect of extracts derived from F\&V or, for example, mouthwash enriched with F\&V extracts. Moreover, only papers in English were included. Due to the potential erosive effect of fruit juice, ${ }^{16}$ studies on fruit juice intake were also excluded from this systematic review. Different age groups were included in this study and there was no restriction on the severity of periodontitis or gingivitis. The following periodontal outcomes were used to assess the periodontal status: pocket depth/probing pocket depth (PD/PPD), plaque index (PI), bleeding index $(\mathrm{BI})$, bleeding on probing (BOP), alveolar bone loss $(\mathrm{ABL})$, clinical attachment level (CAL), attachment 
loss (AL), gingival cervical fluid (GCF), gingival index (GI), gingival recession (GR), modified gingival index (MGI), community periodontal index of treatment needs/community periodontal index (CPITN/CPI), sulcus bleeding index (SBI), vascular endothelial growth factor (VEGF), and tooth loss (TL). Periodontal disease events noted during the observational period were also used to assess the periodontal status. The concentration of vitamin $\mathrm{C}$ in blood serum, ferricreducing antioxidant power (FRAP) and Trolox equivalent antioxidant capacity (TEAC) were determined. For evaluating the inflammation status, the concentration of interleukin $1 \beta$ (IL-1 $\beta$ ) and interleukin 6 (IL-6) was measured. All the papers included in references were additionally reviewed, but there was only 1 report that met all the inclusion criteria. The literature search is illustrated in Fig. 1.

\section{Analysis of study bias and quality}

To assess the potential sources of bias, the studies were reviewed using the critical appraisal checklists from the Scottish Intercollegiate Guideline Network. ${ }^{17}$ Analysis of the quality of the RCTs was independently performed by 2 investigators (ASR, KM), using a 9-category scoring system, being a modified version of the system proposed by Downs and Black. ${ }^{18}$ The cohort and cross-sectional studies were also assessed by 2 reviewers (ASR, KM) in terms of their methodological quality, using an adapted Newcastle-Ottawa Quality Assessment Scale for cohort studies ${ }^{19}$ and a separate scale for cross-sectional studies. ${ }^{20}$

\section{Data extraction}

Data items extracted from the included papers were as follows: author, year, country, study design, study duration, sample size, participant characteristics (age, gender and periodontal status), method(s) for assessing F\&V intake, periodontal status indicator(s), intervention (I) and control (C) conditions in RCTs, and results reported for periodontal status indicators. The data was extracted independently by 2 authors (ASR, KM), with any conflicting judgments discussed and a $3^{\text {rd }}$ independent judgment (JB) sought if agreement could not be reached.

\section{Search results}

In total, electronic search through the 3 databases and references revealed 181 potentially relevant papers. Seventy-five papers were selected from the MEDLINE (PubMed) database, 55 from the Scopus database, 50 from the Google Scholar database, and 1 from the reference lists. During the first screening, based on title and abstract, 120 records were excluded from future analysis. In addition, 12 studies were excluded from analysis due to duplication. In the next step, 34 full-text articles failed to meet the inclusion criteria. Overall, a total of 15 articles were enrolled into the qualitative synthesis and systematic review.

\section{Quality assessment}

Four of the studies examining dietary interventions obtained an overall average rating of $88 \%$, which allowed these studies to be classified as high-quality. ${ }^{21}$ For example, the study by Staudte et al. met 7 out of 9 conditions and obtained a total score of 77.77, and was qualified as a high-quality study. ${ }^{22}$ In the case of all the assessed observational studies, sample representativeness was considered to be adequate and the presence of periodontal diseases was confirmed by clinical indicators reported at baseline. The comparability of the study groups based on age, gender and socioeconomic factors was described. Follow-up and outcome assessment was judged to be adequate in all studies. Only 1 study did not describe the timeline of the research. ${ }^{23}$ Sample size or power calculations were reported in 5 studies. ${ }^{24-28}$ Periodontal therapy was provided by a range of operators, including specialists, general dentists and hygienists. Overall, the methodological quality of all the observational studies was considered to be medium.

\section{Study characteristics}

The total number of subjects participating in the studies selected for review was 10,604 . The age of the participants in the studies ranged from 15 to 90 years. One study was conducted with children and teenagers,$^{29} 15$ studies involved adults, ${ }^{22-36}$ while older adults ( $\geq 65$ years) participated in 9 studies. $^{22,24,25,27,28,31-33,36}$ One study included pregnant women. ${ }^{35}$ Among the studies selected for review, 4 involved dietary intervention experiments, ${ }^{22,32-34} 1$ was a retrospective cohort study, ${ }^{26} 2$ were prospective cohort studies, ${ }^{24,36}$ and 8 were cross-sectional studies. ${ }^{23,25,27-31,35}$ The consumption of F\&V was mostly assessed using the Food Frequency Questionnaire (FFQ) method, ${ }^{26,28,30,33,35,36}$ the food diary method ${ }^{33}$ and the 24-hour recall method. ${ }^{26-28,30,31}$ We abstracted the following data from each study: author's first name, year of publication, country where the study was conducted, study design, study duration, sample size, characteristics of the participants, use of periodontal indices, and results. The studies selected for review are characterized in Table 1. 


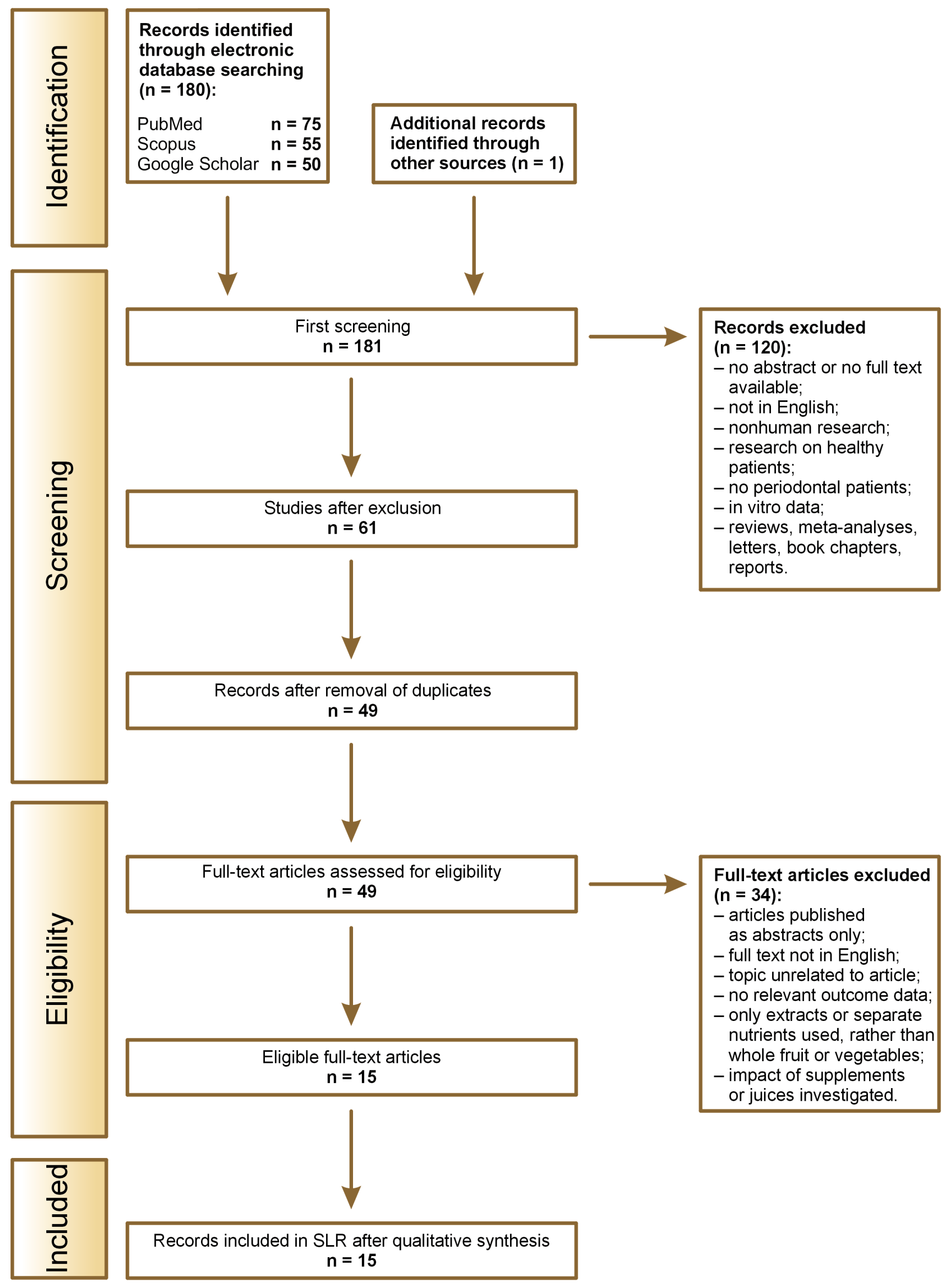

Fig. 1. Flow chart of the literature review process

SLR - systematic literature review. 


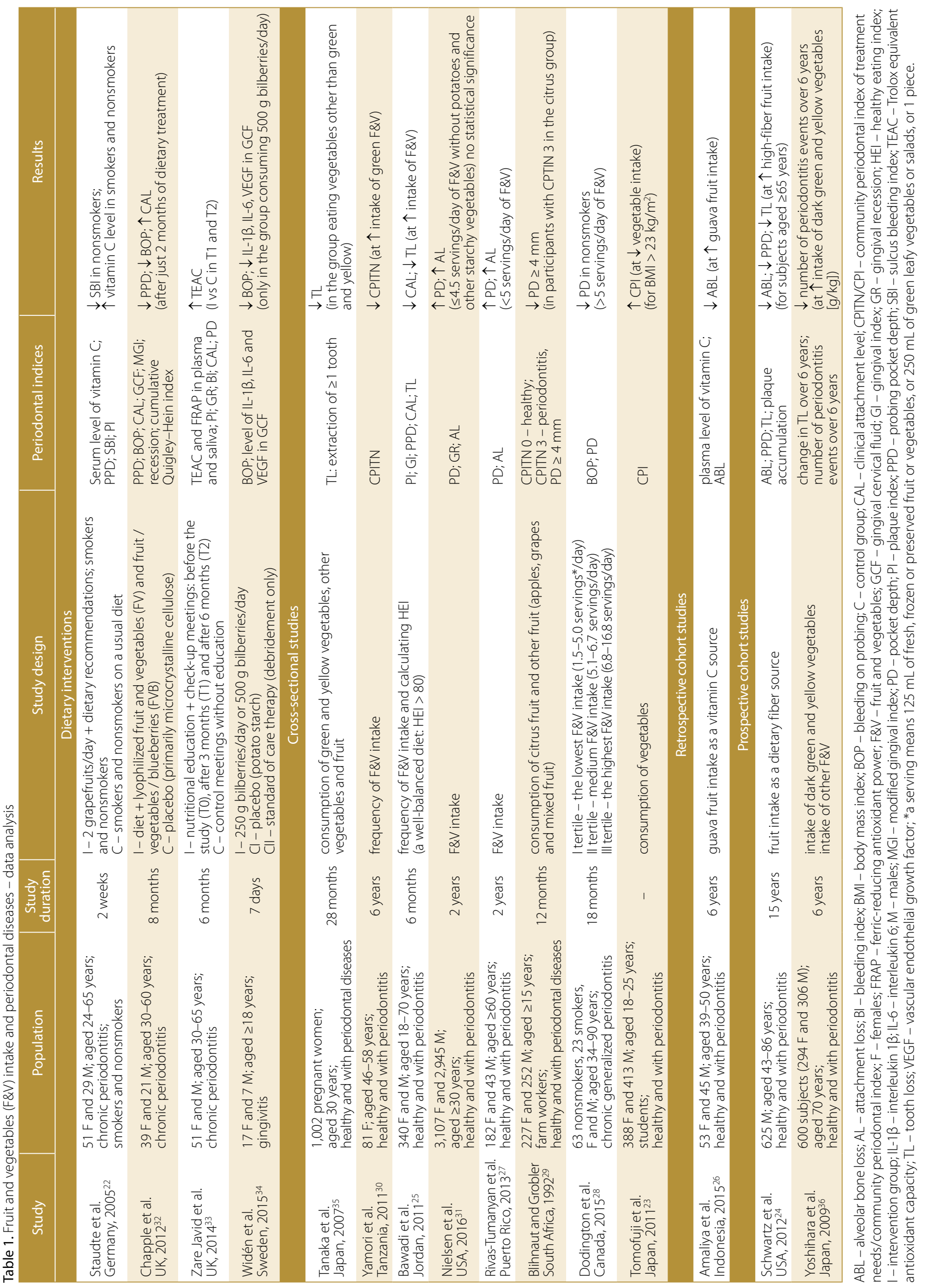




\section{Fruit and vegetables consumption as an adjunct to standard of care treatment (dietary intervention studies)}

Although diet is described as a modifiable risk factor for periodontal diseases, ${ }^{37}$ data on dietary manipulations where F\&V were added to the diet of patients with periodontal diseases is very limited. Our review found 4 such dietary intervention studies. ${ }^{22,32-34}$ In the study by Staudte et al., it was found that incorporation of 2 servings of grapefruit (500 g) into the diet of smoking and nonsmoking patients diagnosed with chronic periodontitis resulted in a significant increase in blood serum vitamin $C$ concentration after a 2-week intervention in comparison to a control group consuming a usual diet. ${ }^{22}$ Moreover, among diseased nonsmokers, a significant reduction in the value of SBI was also observed. ${ }^{22}$ Widén et al. reported a significant reduction in BOP, as well as in the concentrations of proinflammatory cytokines (IL-1 $\beta$ and IL-6) and VEGF in GCF among individuals with gingivitis who consumed $500 \mathrm{~g}$ bilberries per week in comparison to controls receiving a placebo. ${ }^{34}$ Moreover, it was stated that the intake of $500 \mathrm{~g}$ bilberries caused similar changes in BOP as did standard of care therapy (debridement only). ${ }^{34}$ Significant improvements in the PPD and BOP values were observed after only 2 months in patients suffering from chronic periodontitis who received nonsurgical treatment and freeze-dried F\&V..$^{32}$ In a 6-month study conducted by Zare Javid et al. among adults suffering from chronic periodontitis who had completed nonsurgical treatment, nutritional education activities focusing on adequate intake of $F \& V$ ( $~ \geq 5$ servings daily) were linked with a significant increase in plasma total antioxidant capacity, as measured by the TEAC assay, compared to the control group. ${ }^{33}$ No significant differences were observed between the groups with respect to the periodontal indices measured (PI, GR, BI, CAL, and PD) or the FRAP index. ${ }^{33}$ To conclude, on the basis of the collected dietary intervention studies, it can be stated that the incorporation of F\&V into the diet, especially citrus fruit, berries and lyophilized $\mathrm{F} \& \mathrm{~V}$, as well as nutritional education activities focusing on adequate $F \& V$ intake, may be useful as an adjunctive measure in the management of periodontitis or gingivitis. However, further RCTs are required to confirm this effect.

\section{Fruit and vegetable intake in the prevention of periodontal diseases (cohort and cross- sectional studies)}

We saw an association between F\&V intake and periodontal health in 8 cross-sectional studies and 3 cohort studies. For example, Yamori et al., in a study of 81 women aged 46-58 years, reported that the severity of periodontitis (assessed on the basis of the CPITN) was inversely correlated with the frequency of intake of green $F \& V^{30}$ Bawadi et al. stressed that a well-balanced diet (as assessed by the healthy eating index, HEI > 80) with adequate intake of F\&V (min 5 servings per day) was associated with improved CAL. ${ }^{25}$ It was also observed that higher intake of F\&V was related to a lower number of lost teeth. ${ }^{25}$ In a study conducted by Rivas-Tumanyan et al., it was shown that among elderly patients, having at least 2 teeth with the AL score of $\geq 6 \mathrm{~mm}$ and at least 1 tooth with the PD value of $\geq 5 \mathrm{~mm}$ was significantly associated with low intake of $F \& V\left(<5\right.$ servings per day). ${ }^{27}$ In a study conducted during the years 2009-2010 and 2011-2012 on a population of 6,052 adults, Nielsen et al. observed that insufficient intake of $F \& V$ of $\leq 4.5$ servings per day was associated with an increase in PD and AL; however, this relation was statistically insignificant. ${ }^{31}$ Blihnaut and Grobler, in a study of 479 fruit pickers in South Africa, assessed the relationship between periodontal health (evaluated on the basis of CPITN) and the intake of fresh fruit (including citrus fruit, apples, grapes, and mixed fruit). ${ }^{29}$ The recommended daily fruit intake was established as at least 8 apples, 8 bunches of grapes, 8 oranges or mandarins, or 11 fruits being a mixture of different kinds. In individuals eating citrus fruit, $\mathrm{PD} \geq 4 \mathrm{~mm}$ was diagnosed less often. ${ }^{29}$ Similarly, during an 18-month observation, Dodington et al. recorded that increased intake of $F \& V$ ( $\geq 5$ servings per day) was associated with a significantly lower incidence of PD $>3 \mathrm{~mm} .{ }^{28}$ However, this association was seen only among nonsmokers. Tomofuji et al. observed that the frequency of vegetable consumption among obese students diagnosed with periodontitis was significantly lower (10.9\%) than among non-obese individuals (33\%). ${ }^{23}$ No such association was found in underweight students and students with normal body weight. ${ }^{23}$ In a study conducted by Tanaka et al., involving 1,002 pregnant women, a relationship between the intake of F\&V and TL was seen. ${ }^{35}$ The lowest number of lost teeth was recorded in the group of women consuming the largest amounts ( $>170$ g per day) of vegetables other than green or yellow, such as cabbage, radishes and onions. ${ }^{35}$ The protective role of adequate $F \& V$ intake on periodontal health, as reported by cross-sectional studies, was also confirmed in 2 prospective and 1 retrospective cohort studies. For example, Schwartz et al. observed among veterans aged over 65 years that the intake of high-fiber fruits, such as bananas, apples, pears, oranges, and plums, was associated with a lower risk of progression in ABL, PPD and TL. ${ }^{24}$ No significant associations were seen in younger veterans. ${ }^{24}$ Moreover, the intake of dark green and yellow vegetables was negatively correlated with the number of periodontitis events during the 6 years of observations conducted in a group of 600 older adults. ${ }^{36}$ In her retrospective study, Amaliya et al. found opposite associations between the consumption of guava fruit, rich in vitamin $C$, 
and the amount of ABL. ${ }^{26}$ To conclude, the findings from the collected studies suggest strong inverse associations between the progression of periodontal diseases, especially periodontitis, and adequate consumption (at least 5 servings a day) of F\&V. In this matter, special attention should be given to adequate consumption of dark green and yellow vegetables (spinach, broccoli, yellow peppers, and carrots) and some other vegetables (cabbage, radishes and onions), and fruits with a high content of vitamin $\mathrm{C}$ (black currants, grapefruits and oranges) and dietary fiber (bananas, apples, pears, berries, and plums).

\section{Discussion}

The data collected from the cross-sectional studies and confirmed by the prospective and retrospective cohort studies clearly indicates that the recommended intake of $F \& V$ (at least 5 servings a day) is a significant factor that can prevent the progression of periodontal diseases and even tooth loss among people at all ages. It should be highlighted, however, that smoking is a significant risk factor in the progression of periodontitis, irrespective of the actual intake of F\&V. ${ }^{37}$ Our findings were similar to those presented by Nanri et al. and Moynihan, where higher F\&V consumption was also associated with better oral health-related quality of life. ${ }^{38,39}$ Moreover, our systematic literature review indicated that incorporating F\&V (especially citrus fruit and berries) into the diet, as well as nutritional education activities that stress the need for adequate intake of $F \& V$ by individuals with various forms of periodontal diseases, could lead to a significant enhancement in the oral health status by improving gingival inflammation indices ${ }^{34}$ and inhibiting the progression of periodontitis. ${ }^{22,33,34} \mathrm{An}$ interesting alternative seems to be provided by the potential use of freeze-dried F\&V in the treatment of periodontitis. ${ }^{32}$ This particularly pertains to individuals who, due to the progression of periodontitis and disturbed mastication (i.e., older adults) do not consume adequate amounts of fresh F\&V. It has been stated that almost $85 \%$ of patients currently reporting periodontitis are 65 years or older, ${ }^{40}$ which is due to the fact that the number of teeth decreases with age and the remaining teeth get more exposed and sensitive to pathogenic agents. Periodontitis leads to difficulties in the consumption of certain types of foods, especially raw F\&V, which are hard to chew. Therefore, in the case of such people, lyophilized F\&V products could constitute an interesting approach to the prevention of periodontal diseases, and these products could be recommended as safe adjuncts to nonsurgical periodontal therapy in patients with gingivitis and periodontitis.

Fruit and vegetables are a good source of multiple nutrients, including vitamins $\mathrm{A}, \mathrm{E}$ and $\mathrm{C}$, and phytochemicals, as well as dietary fiber. All these compounds can affect periodontal health, as they function as antioxidants, antibacterial agents (inhibiting the adhesion of such pathogens as Streptococcus mutans to plaque) and enhancers of cell-mediated immunity. ${ }^{32,41,42}$ For example, grapefruits are among the richest dietary sources of vitamin C. ${ }^{39}$ Two portions of grapefruit provide about $240 \mathrm{mg}$ of vitamin C. ${ }^{34}$ The mechanism of action of vitamin $C$ is associated with its antioxidant properties, which help to reduce the production of reactive oxygen radicals, formed during inflammatory processes. ${ }^{39,43}$ Staudte et al. stress that adequate intake of vitamin $C$ is crucial in collagen synthesis and in preventing excessive permeability of the gingival tissue. ${ }^{22}$

Berry fruits, such as cranberries, bilberries and blueberries, are also a significant source of flavanols, anthocyanins and proanthocyanidins. These compounds inhibit the formation of biofilm on tooth surfaces, at the same time exhibiting strong antioxidant and anti-inflammatory activity. ${ }^{34}$ Yellow and dark green vegetables may also have beneficial effects on periodontal health, mostly because they are a source of antioxidants and may lower $\mathrm{pH}$ levels, thus reducing the number of pathogens. ${ }^{36}$ Lectins, prostaglandins and thiosulfates contained in onions have antiinflammatory and antibacterial properties. ${ }^{44,45}$ Lupenol present in white cabbage exhibits anti-inflammatory activity. ${ }^{46,47}$ Radish is a good source of glucosinolates, which have antioxidant properties. ${ }^{48} \mathrm{~A}$ number of mechanisms have been proposed to explain the relationship between the intake of high-fiber food and periodontal diseases. The protective effect of fiber consists in controlling serum glucose levels and insulin resistance, since diabetes has been unequivocally confirmed as a major risk factor for periodontitis. ${ }^{24}$ Moreover, high-fiber foods mechanically remove plaque from tooth surfaces. ${ }^{49}$

Our study has several strengths. Our systematic review was based on 8 cross-sectional studies, as well as on 2 prospective studies and 1 retrospective cohort study, all of them conducted on various populations, which allowed us to conclude that higher intake of $F \& V$ is positively associated with the periodontal health status. However, this systematic review also has several limitations. Firstly, the studies included in the systematic review used different periodontal disease indices, which made the results of those studies difficult to compare. Secondly, different studies used different methods of dietary assessment, such as 24-hour recall, food diaries and FFQ. Moreover, in the cohort and cross-sectional studies, either the frequency of $F \& V$ intake or the consumption of various fruit (e.g., rich in vitamin $C$ and dietary fiber) and vegetables (e.g., dark green and yellow) was evaluated. Thirdly, there were differences in the classification of F\&V across studies. Types of consumed F\&V differed according to geographical locations. These factors could affect our results. Fourthly, there were only a few dietary intervention studies that might confirm the effect of $F \& V$ as an adjunctive measure in the management of periodontal diseases. Therefore, more high-quality dietary intervention studies, 
focusing on different age groups, but with special attention paid to older age groups, are required to confirm our findings. Dietary advice for the prevention of oral diseases also has to be a part of routine patient education practices. However, we should also highlight that there is growing awareness among dental professionals of the beneficial effects of adequate diets on the periodontal tissue condition. According to Kelly and Moynihan, $66 \%$ of the surveyed dentists stated that an adequate, well-balanced diet significantly affected the progression of periodontal diseases. ${ }^{50}$ Most respondents declared that the intake of vitamin $\mathrm{C}$ was significantly associated with improved periodontal health (70.2\%), with the recommended level of F\&V intake ranking $2^{\text {nd }}(64.3 \%)$.

This systematic review suggests that the consumption of $F \& V$, at least 5 servings per day, especially dark green and yellow vegetables (spinach, broccoli, yellow peppers, and carrots) and some other vegetables (cabbage, radishes, onions), as well as fruit rich in vitamin C (black currants, grapefruits, oranges) and dietary fiber (bananas, apples, pears, berries, and plums) may prevent the progression of periodontal diseases, especially periodontitis, and even tooth loss. Moreover, incorporation of specific F\&V (berries, citrus fruit) into the diet or, alternatively, their lyophilized forms, and nutritional education activities seem to support the standard of care therapy of gingivitis and periodontitis. However, further observational and well-designed experimental studies, with homogeneous periodontal status outcomes, are needed to confirm these findings. Furthermore, professional dietetics care for periodontal patients should be an integral component of the healthcare program.

\section{References}

1. Woźniewicz M, Nowaczyk PM, Kurhańska-Flisykowska A, et al. Consumption of cranberry functional beverage reduces gingival index and plaque index in patients with gingivitis. Nutr Res. 2018;58:36-45.

2. Mariotti A, Hefti AF. Defining periodontal health. BMC Oral Health. 2015;15(Suppl 1):S6.

3. Jin LJ, Lamster IB, Greenspan JS, Pitts NB, Scully C, Warnakulasuriya S. Global burden of oral diseases: Emerging concepts, management and interplay with systemic health. Oral Dis. 2016;22:609-619.

4. Caton JG, Armitage G, Berglundh T, et al. A new classification scheme for periodontal and peri-implant diseases and conditions - introduction and key changes from the 1999 classification. J Periodontol. 2018;89(Suppl 1):1-8.

5. Marsh PD, Bradshaw DJ. Dental plaque as a biofilm. J Ind Microbiol. 1995;15:169-175.

6. Yang H, Xiao L, Zhang L, Deepal S, Ye G, Zhanga X. Epidemic trend of periodontal disease in elderly Chinese population, 1987-2015: A systematic review and meta-analysis. Sci Rep. 2017;7:45000.

7. Iwata $T$, Yamato M, Ishikawa I, Ando T, Okano T. Tissue engineering in periodontal tissue. Anat Rec (Hoboken). 2014;297:16-25.

8. Pihlstrom BL, Michalowicz BS, Johnson NW. Periodontal diseases. Lancet. 2005;366:1809-1820.

9. Dahiya P, Kamal R, Gupta R. Obesity, periodontal and general health: Relationship and management. Indian J Endocr Metab. 2012;16:88-93.

10. Patel R. The state of oral health in Europe. Report commissioned by the Platform for Better Oral Health in Europe. 2012. http://www. oralhealthplatform.eu/wp-content/uploads/2015/09/Report-theState-of-Oral-Health-in-Europe.pdf. Accessed February 25, 2018.
11. Palmer CA. Important relationships between diet, nutrition, and oral health. Nutr Clin Care. 2001;4:4-14.

12. Moynihan PJ. The role of diet and nutrition in the etiology and prevention of oral diseases. Bull World Health Organ. 2005;83:694-699.

13. Van der Putten GJ, Vanobbergen J, De Visschere L, Schols J, de Baat C. Association of some specific nutrient deficiencies with periodontal disease in elderly people: A systematic literature review. Nutrition. 2009;25:717-722.

14. Small L, Anderson, D, Melnyk, BM. Prevention and early treatment of overweight and obesity in young children: A critical review and appraisal of the evidence. Pediatr Nurs. 2007;33:127,149-152,155-161.

15. Shamseer L, Moher D, Clarke M, et al.; PRISMA-P Group. Preferred reporting items for systematic review and meta-analysis protocols (PRISMA-P) 2015: Elaboration and explanation. BMJ. 2015;350:g7647.

16. Zimmer S, Kirchner G, Bizhang M, Benedix M. Influence of various acidic beverages on tooth erosion. Evaluation by a new method. PLOS ONE. 2015;10:e0129462.

17. Scottish Intercollegiate Guidelines Network. Critical appraisal notes and checklists. http://sign.ac.uk/methodology/checklists. html. Accessed February 25, 2018.

18. Downs $\mathrm{SH}$, Black N. The feasibility of creating a checklist for the assessment of the methodological quality both of randomised and non-randomised studies of health care interventions. J Epidemiol Community Health. 1998;52:377-384.

19. Wells GA, Shea B, O'Connell D, et al. The Newcastle-Ottawa Scale (NOS) for assessing the quality of nonrandomised studies in meta-analyses. 2008. http://www.ohri.ca/programs/clinical_epidemiology/nosgen.pdf. Accessed February 25, 2018.

20. Modesti PA, Reboldi G, Cappuccio FP, et al.; ESH Working Group on CV Risk in Low Resource Settings. Panethnic differences in blood pressure in Europe: A systematic review and meta-analysis. PLoS ONE. 2016;11:e0147601.

21. Wickham CA, Carbone ET. Who's calling for weight loss? A systematic review of mobile phone weight loss programs for adolescents. Nutr Rev. 2015;73:386-398.

22. Staudte H, Sigush BW, Glockmann E. Grapefruit consumption improves vitamin C status in periodontitis patients. Br Dent J. 2005;199:213-217.

23. Tomofuji T, Furuta M, Ekuni D, et al. Relationships between eating habits and periodontal condition in university students. J Periodontol. 2011;82:1642-1649.

24. Schwartz N, Kaye EK, Nunn ME, Spiro A, Garcia RI. High-fiber foods reduce periodontal disease progression in men aged 65 and older: the Veterans Affairs normative aging study/dental longitudinal study. J Am Geriatr Soc. 2012;60:676-683.

25. Bawadi HA, Khader YS, Haroun TF, Al-Omari M, Tayyem RF. The association between periodontal disease, physical activity and healthy diet among adults in Jordan. J Periodontal Res. 2011;46:74-81.

26. Amaliya A, Laine ML, Delanghe JR, Loos BG, Van Wijk AJ, Van der Velden U. Java project on periodontal diseases: Periodontal bone loss in relation to environmental and systemic conditions. J Clin Periodontol. 2015;42:325-332.

27. Rivas-Tumanyan S, Campos M, Zevallos JC, Joshipura KJ. Periodontal disease, hypertension, and blood pressure among older adults in Puerto Rico. J Periodontol. 2013;84:203-211.

28. Dodington DW, Fritz PC, Sullivan PJ, Ward WE. Higher intakes of fruits and vegetables, $\beta$-carotene, vitamin $C$, $a$-tocopherol, EPA, and DHA are positively associated with periodontal healing after nonsurgical periodontal therapy in nonsmokers but not in smokers. J Nutr. 2015;145:2512-2519.

29. Blihnaut JB, Grobler SR. High fruit consumption and the periodontal status of farm workers. Clin Prev Dent. 1992;14:25-28.

30. Yamori M, Njelekela M, Mtabaji J, Yamori Y, Bessho K. Hypertension, periodontal disease, and potassium intake in nonsmoking, nondrinker African women on no medication. Int J Hypertens. 2011;2011:695719.

31. Nielsen SJ, Trak-Fellermeier MA, Joshipura K, Dye BA. Dietary fiber intake is inversely associated with periodontal disease among US adults. J Nutr. 2016;146:2530-2536.

32. Chapple ILC, Milward MR, Ling-Mountford N, et al. Adjunctive daily supplementation with encapsulated fruit, vegetable and berry juice powder concentrates and clinical periodontal outcomes: A double-blind RCT. J Clin Periodontol. 2012;39:62-72. 
33. Zare Javid A, Seal CJ, Heasman P, Moynihan PJ. Impact of a customized dietary intervention on antioxidant status, dietary intakes and periodontal indices in patients with adult periodontitis. $J$ Hum Nutr Diet. 2014;27:523-532.

34. Widén $C$, Coleman $M$, Cariten $S$, Karlgren-Andersson $P$, Renvert $S$, Persson GR. Consumption of bilberries controls gingival inflammation. Int J Mol Sci. 2015;16:10665-10673.

35. Tanaka K, Miyake Y, Sasaki S, et al. Relationship between intake of vegetables, fruit and grains and the prevalence of tooth loss in Japanese women. J Nutr Sci Vitaminol (Tokyo). 2007;53:522-528.

36. Yoshihara A, Watanabe R, Handa N, Miyazaki H. A longitudinal study of the relationship between diet intake and dental caries and periodontal disease in elderly Japanese subjects. Gerodontol. 2009;26:130-136.

37. AlJehani YA. Risk factors of periodontal disease: Review of the literature. Int J Dent. 2014;2014:182513.

38. Nanri H, Yamada $Y$, Itoi A, et al. Frequency of fruit and vegetable consumption and the oral health-related quality of life among Japanese elderly: A cross-sectional study from the Kyoto-Kameoka Study. Nutrient. 2017;9:e1362.

39. Moynihan PJ. The role of diet and nutrition in the etiology and prevention of oral diseases. Bull World Health Organ. 2005;83:694-699.

40. White D, Tsakos G, Pitts NB, et al. Adult Dental Health Survey 2009: Common oral health conditions and their impact on the population. Br Dent J. 2000;213:567-572.

41. La VD, Howell AB, Grenier D. Cranberry proanthocyanidins inhibit MMP production and activity. J Dent Res. 2009;88:627-632.

42. Bonifait L, Grenier D. Cranberry polyphenols: Potential benefits for dental caries and periodontal disease. J Can Dent Assoc. 2010;76:a130.

43. Iwasaki M, Moynihan $P$, Manz MC, et al. Dietary antioxidants and periodontal disease in community-based older Japanese: A 2-year follow-up study. Public Health Nutr. 2013;16:330-338.

44. Wilson EA, Demming-Adams B. Antioxidant, anti-inflammatory, and antimicrobial properties of garlic and onions. Nutr Food Sci. 2007;37:178-183.

45. Corzo-Martinez M, Corzo N, Villamiel M. Biological properties of onions and garlic. Trends Food Sci Tech. 2007;18:609-625.

46. Lin JY, Li CY, Hwang IF. Characterization of the pigment components in red cabbage (Brassica oleracea L. var.) juice and their antiinflammatory effects on LPS-stimulated murine splenocytes. Food Chem. 2008;109:771-781.

47. Saleem M. Lupeol, a novel anti-inflammatory and anti-cancer dietary triterpene. Cancer Lett. 2009;285:109-115.

48. Lugasi A, Dworschak E, Blazovics A, Kocsis I, Kéry A. Antioxidant and free radical scavenging properties of squeezed juice from black radish (Raphanus sativus L. var. niger) root. Phytother Res. 1998;12:502-506.

49. Kondo K, Ishikado A, Morino K, et al. A high-fiber, low-fat diet improves periodontal disease markers in high-risk subjects: A pilot study. Nutr Res. 2014;34:491-498.

50. Kelly SA, Moynihan PJ. Attitudes and practices of dentists with respect to nutrition and periodontal health. Br Dent J. 2008;205:E9. 
